\title{
Associações entre mobilidade corporal, autoimagem e qualidade de vida em indivíduos idosos integrantes de um programa sistemático de atividades físicas
}

\author{
Dirceu Ribeiro Nogueira da Gama; Maria Izabel Ferreira Batista"; Ben Hur Soares"*; Nádia Souza Lima da \\ Silva"**; Rodrigo Gomes de Souza Vale"***
}

\section{Resumo}

O objetivo do presente estudo foi investigar as associações entre mobilidade física, imagens corporais e percepção de qualidade de vida em idosos integrantes de um programa sistemático de atividades físicas. A amostra consistiu de 28 idosos de ambos os sexos (16 homens e 12 mulheres). Os instrumentos para a coleta de dados foram o teste "8 foot up-and-go" (FUG), o questionário "Minha imagem corporal" e o questionário WHOQOL-Old para Qualidade de Vida. Os dados foram tratados por meio da estatística descritiva e correlacional. O teste de correlação de Spearman mostrou uma correlação positiva entre a idade dos idosos e o escore do teste FUG $(p<0,001)$. Também foram detectadas correlações positivas e significativas entre a percepção de condição física e as facetas Fac 1, Fac 2 e Fac 3 do WHOQOL-OLD ( $p$ $<0,05)$; Fac 6 e idade $(p<0,05)$; escore do teste FUG e as Fac 5 e Fac $6(p<0,05)$; percepção de saúde e Fac $4(p<0,05)$. Houve correlações negativas e significativas entre idade e as percepções de condição física e de habilidade corporal ( $p<0,05)$; escore do teste FUG e as percepção de condição física e de habilidade corporal $(p<$ $0,05)$. O estudo permitiu concluir que os retardos na capacidade motora decorrentes do avanço da idade estão associados à redução da condição física e da habilidade corporal, assim como a percepção do idoso sobre o condicionamento físico, a sua mobilidade corporal e a idade cronológica pode interferir nas facetas da qualidade de vida, porém, em diferentes proporções.

* Educador físico. Doutorado em Filosofia pela Universidade Gama Filho, Rio de Janeiro/RJ. Programa de Pós-Graduação em Ciências do Exercício e do Esporte do Instituto de Educação Física e Desportos da Universidade do Estado do Rio de Janeiro, Rio de Janeiro/RJ. E-mail: paula.dirceu@hotmail.com.

** Educadora física. Mestranda do Programa de Pós-Graduação em Ciências do Exercício e do Esporte da Universidade do Estado do Rio de Janeiro, Rio de Janeiro/RJ. E-mail: mariaizabelfbatista@hotmail.com.

*** Educador físico. Mestrado em Envelhecimento Humano pela Universidade de Passo Fundo, Passo Fundo/ RS. Doutorando do Programa de Pós-Graduação em Envelhecimento Humano da Universidade de Passo Fundo, Passo Fundo/RS. Faculdade de Educação Física e Fisioterapia da Universidade de Passo Fundo, Passo Fundo/RS. E-mail: benhur@upf.br

****** Educadora física. Pós-doutorado em Ciências da Saúde pela Universidade do Porto/Portugal. Doutorado em Educação Física pela Universidade Gama Filho, Rio de Janeiro/RJ. Programa de Pós-Graduação em Ciências do Exercício e do Esporte do Instituto de Educação Física e Desportos da Universidade do Estado do Rio de Janeiro, Rio de Janeiro/RJ. E-mail: nadiaslimas@gmail.com.

***** Pós-doutorado em Biociências pela Universidade Federal do Estado do Rio de Janeiro, Rio de Janeiro/RJ. Doutorado em Ciências da Saúde pela Universidade Federal do Rio Grande do Norte, Natal/RN. Programa de Pós-Graduação em Ciências do Exercício e do Esporte do Instituto de Educação Física e Desportos da Universidade do Estado do Rio de Janeiro Rio de Janeiro/RJ. Curso de Educação Física da Universidade Estácio de Sá, Cabo Frio/RJ. E-mail: rodrigovale@globo.com.

$\rightarrow$ http://dx.doi.org/10.5335/rbceh.v16i3.10029 
Palavras-Chave: Idosos. Mobilidade corporal. Autoimagem. Qualidade de vida. Atividade física.

\section{Introdução}

O envelhecimento consiste em um processo biopsíquico caracterizado pelo declínio das funções sensoriais, motoras e mentais, recebendo a influência tanto de fatores genéticos como dos hábitos de vida (SANTOS et al., 2009). No Brasil, ao longo dos últimos 40 anos, os censos demográficos registraram um paulatino crescimento da população idosa. Estima-se que, ao longo da última década, o contingente composto por pessoas com 65 anos ou mais tenha se tornado $7,4 \%$ do total de brasileiros (INOUYE et al., 2018).

A deflagração do processo de envelhecimento faz com que o indivíduo passe a sentir dificuldades progressivas na efetivação de cálculos aritméticos, gestão de recursos financeiros, articulação verbal de pensamentos, leitura e interpretação de textos, manutenção de relacionamentos afetivos e execução de ações motoras finas e grossas como escrever, caminhar, subir e descer escadas (OSWALD et al., 2006). A intensidade dessas perdas tende a diminuir a qualidade de vida $(\mathrm{QV})$ do idoso por implicar em comprometimentos na realização de atividades de vida diária (AVD) com independência (VALE et al., 2018).

A Organização Mundial de Saúde considera QV como a percepção que o indivíduo tem de sua posição na vida, no contexto da cultura e no sistema de valores em que vive, e em relação a seus objetivos, expectativas, padrões e preocupações (FLECK et al., 2003). Por sua vez, a elaboração de tal percepção recebe a influência da saúde física, crenças pessoais, estado psicológico e relações socioambientais (FUNG, 2013). Portanto, a QV remete a uma interação dinâmica das condições externas que balizam a vida do indivíduo com a compreensão dele acerca dessas condições (HURD-CLARKE, 2001).

Em função da pluridimensionalidade, a liberdade e a autonomia motoras afetam diretamente a $\mathrm{QV}$, pois movimentar-se com autocontrole e voluntariedade são fatores relevantes para a manutenção de uma existência digna e emancipada (ZIELI $\square$ SKA-WI $\square$ CZKOWSKA, 2017). A prática regular e sistemática de atividades físicas pode minimizar os comprometimentos motores ocasionados pelo envelhecimento, fazendo com que a prática regular de exercícios físicos seja considerada uma estratégia eficiente para o incremento da QV no plano pessoal (VALE et al., 2018). No que compete aos seus conteúdos, as combinações sistemáticas e regulares de exercícios resistidos, de flexibilidade e aeróbios são capazes de exercer efeitos positivos sobre os equilíbrios estático e dinâmico, força muscular, tempo de reação, velocidade, resistência, percepção espaço-temporal e ritmo (CHAO et al., 2015; CHAN et al., 2015; PITKÄLÄ et al., 2013; LAM et al., 2013). A preservação destas valências é importante para que o idoso consiga, por exemplo, caminhar, inclinar-se, correr, subir e descer escadas, empunhar obje- 
tos, transportar cargas, sentar e levantar o corpo com segurança e voluntariedade (CHAN et al., 2015; SOÓSOVÁ, 2016; TAIROVA; LORENZ, 2011).

Por outro lado, a consciência de mover o corpo com integridade, fluência e eficiência interfere no conjunto de representações mentais que o sujeito constrói de si mesmo no transcorrer de sua existência cotidiana, seja ele idoso ou não (TYLKA; WOOD-BARCALOW, 2015). Estas representações recebem o nome de imagem corporal ou autoimagem (WEBB; WOOD-BARCALOW; TYLKA, 2015). Uma mobilidade física ampla e voluntariamente controlada condiciona a produção de expectativas positivas sobre os aspectos funcionais do próprio corpo, ou seja, redunda na estruturação de imagens corporais satisfatórias (TYLKA; WOOD-BARCALOW, 2015). Isso significa que as avaliações que uma pessoa faz de sua existência sofrem influência do quanto ela se reconhece nos movimentos corporais que consegue executar. Caso ela não se identifique com as suas capacidades motoras, possivelmente estas avaliações poderão receber um significado negativo (WEBB; WOOD-BARCALOW; TYLKA, 2015).

Sendo assim, as informações anteriormente expostas permitem supor que a prática sistemática de exercícios é capaz de atenuar os efeitos deletérios do processo de envelhecimento em relação a mobilidade corporal e também impactar de maneira positiva a constituição das autoimagens de indivíduos idosos, assim como as suas percepções sobre a QV. Dessa forma, o objetivo do presente estudo foi investigar as associações entre mobilidade física, imagens corporais e percepção de $\mathrm{QV}$ em idosos integrantes de um programa sistemático de atividades físicas.

\section{Materiais e métodos}

A presente pesquisa é do tipo descritiva correlacional e transversal, em que a população do estudo foi de 42 idosos de ambos os sexos, com idades entre 66 e 91 anos de idade, participantes de um programa de atividade física vinculado a uma universidade pública situada no Estado do Rio de Janeiro. Como critérios de inclusão, optou-se pelos indivíduos com idade igual ou superior a 60 anos; independentes em suas atividades de vida diária; participantes regulares do programa há pelo menos quatro meses e considerados clinicamente aptos para realizarem atividades físicas regulares conforme avaliação médica. Foram excluídos aqueles que faltaram $20 \%$ ou mais das sessões de treino ministradas neste período; exibiam distúrbios motores em decorrência de cirurgias recentes e apresentavam algum tipo de processo inflamatório. Com base na aplicação desses critérios, 28 idosos de ambos os gêneros (16 homens e 12 mulheres) acabaram sendo selecionados para comporem a amostra.

O estudo foi submetido e aprovado pelo Comitê de Ética Institucional (parecer 1.617.605). Os participantes assinaram um Termo de Consentimento Esclarecido conforme as diretrizes reguladoras de pesquisas com seres humanos 
contidas na Resolução 580/18 do Conselho Nacional de Saúde e na Declaração de Helsinki.

No que diz respeito à coleta de dados, realizou-se inicialmente uma anamnese a fim de se obter informações sobre o estado geral de saúde e a situação econômica e demográfica dos participantes. Após a anamnese, seguiram-se os procedimentos de avaliação da mobilidade física, imagem corporal e qualidade de vida.

Para a avaliação da mobilidade física, utilizou-se o teste 8 foot up-and-go (FUG) (RIKLI; JONES, 1999), que visa averiguar a potência, velocidade, agilidade e equilíbrio dinâmico corporais. $\mathrm{O}$ teste inicia-se com o avaliado sentado em uma cadeira sem braços, que, ao sinal do avaliador deve levantar-se, caminhar o mais rápido possível até um cone posicionado 2,44 metros à frente da cadeira, contorná-lo, retornar ao ponto inicial e sentar novamente na cadeira. $\mathrm{O}$ tempo necessário dispendido na realização da tarefa, medido em segundos e com precisão de até duas casas decimais, constitui o resultado do teste.

Em relação à imagem corporal, aplicou-se o questionário "Minha imagem corporal" (LOVO, 2001). O instrumento é composto de 22 itens agrupados em quatro dimensões: condição física $(\mathrm{CF}$, itens 1 a 4); habilidades corporais (HC, itens 5 a 8); saúde (S, itens 9 a 18) e aparência (A, itens 19 a 22). O respondente avalia cada um dos itens segundo uma escala de 0 a 2 pontos, onde o nível 0 corresponde a "Não Forte", o nível 1 a "Forte" e o nível 2 a "Bastante Forte". O escore do teste varia de zero a 44 pontos, permitindo ao indivíduo fornecer informações acerca de como percebe o seu corpo.

A qualidade de vida foi avaliada recorrendo-se à aplicação individual do questionário WHOQOL-Old de QV (FLECK et al., 2003). Este instrumento baseia-se nos pressupostos de que a $\mathrm{QV}$ é um construto subjetivo, multidimensional e composto por dimensões positivas e negativas, que, por meio de suas 24 questões, avalia seis Facetas, a saber: Faceta 1 - habilidade sensório (FAC1): avalia o funcionamento sensorial e a perda das habilidades sensoriais na qualidade de vida; Faceta 2 - autonomia (FAC2): refere-se a independência na velhice e descreve até que ponto se é capaz de se viver de forma autônoma e tomar suas próprias decisões; Faceta 3 - atividades do passado, presente e futuro (FAC3): descreve a satisfação sobre as conquistas na vida e as coisas a que se anseia; Faceta 4 - participação social (FAC4): delineia a participação em atividades do cotidiano, especialmente na comunidade; Faceta 5 - morte e morrer (FAC5): relaciona-se a preocupações, inquietações e temores sobre a morte e morrer; Faceta 6 - intimidade (FAC6): avalia a capacidade de se ter relações pessoais e íntimas. Cada uma das facetas possui 4 itens em que o escore dos valores possíveis pode variar de 4 a 20 , desde que todos os itens tenham sido preenchidos segundo uma escala numérica que varia de 1 a 5 pontos. Os escores destas seis facetas ou os valores dos 24 itens do módulo WHOQOL-Old podem ser combinados para 
produzir um escore geral (QVG-OLD) para a $\mathrm{QV}$ em adultos idosos.

O programa de intervenção consistiu em três sessões semanais, acontecendo em dias alternados e com duração de 50 minutos. Nas duas primeiras sessões os idosos realizavam exercícios de contrarresistência com halteres, barras, caneleiras e anilhas. Na terceira sessão semanal, faziam atividades multicomponentes em duplas ou em grupos, com foco no treinamento das capacidades coordenativas, utilizando-se bolas, arcos, bastões, cordas e faixas elásticas. Cada sessão foi dividida em parte inicial (10 minutos), parte principal (30 minutos) e parte final (10 minutos).
Os dados foram tratados pelo programa IBM SPSS Statistics 23 e apresentados como média, desvio padrão e valores mínimos e máximos. A normalidade dos dados foi verificada pelo teste de Shapiro-Wilk. O teste de correlação de Spearman foi empregado para analisar as possíveis associações entre as variáveis de estudo. $O$ valor de $p<0,05$ foi adotado para a significância estatística.

\section{Resultados}

A Tabela 1 mostra os valores médios de idade, escores do teste FUG, Condição Física, Habilidade Corporal, Saúde, Aparência, Facetas do WHOQOL-OLD e Escore Total do WHOQOL-OLD.

Tabela 1 - Resultados descritivos de idade, FUG, condição física, habilidade corporal, saúde, aparência, facetas do WHOQOL-OLD e escore total do WHOQOL-OLD.

\begin{tabular}{|l|c|c|c|c|c|}
\hline \multicolumn{1}{|c|}{ Variáveis } & Média & Desvio-padrão & Mínimo & Máximo & $\mathbf{p}$ \\
\hline Idade & 78,00 & 5,45 & 66,00 & 91,00 & 0,901 \\
\hline FUG (segundos) & 7,36 & 1,19 & 5,00 & 9,48 & 0,178 \\
\hline CF & 4,50 & 1,85 & 1,00 & 8,00 & 0,159 \\
\hline HC & 4,00 & 1,87 & 0,00 & 7,00 & 0,056 \\
\hline S & 11,00 & 4,24 & 3,00 & 19,00 & 0,608 \\
\hline A & 6,00 & 1,80 & 2,00 & 8,00 & 0,011 \\
\hline Fac1 & 15,00 & 2,82 & 10,00 & 19,00 & 0,032 \\
\hline Fac2 & 15,50 & 3,15 & 8,00 & 20,00 & 0,434 \\
\hline Fac3 & 14,50 & 2,38 & 11,00 & 20,00 & 0,229 \\
\hline Fac4 & 16,00 & 2,47 & 8,00 & 20,00 & 0,016 \\
\hline Fac5 & 16,00 & 1,72 & 12,00 & 19,00 & 0,195 \\
\hline Fac6 & 15,50 & 2,83 & 10,00 & 20,00 & 0,155 \\
\hline QVG-OLD & 15,08 & 1,79 & 11,83 & 18,50 & 0,394 \\
\hline
\end{tabular}

Nota: DP:; FUG: Teste "8 foot up-and-go"; CF: Condição Física; HC: Habilidade Corporal; S: Saúde; A: Aparência. QVG-OLD: Qualidade de Vida Geral; Fac 1, Fac 2, Fac 3, Fac 4, Fac 5, Fac 6: Facetas do WHOQOL-OLD; Significativo para $p$ $<0,05$. Teste de normalidade de Shapiro-Wilk. 
A Tabela 2 mostra a matriz de correlação de Spearman do teste FUG com a idade e as categorias do teste "Minha imagem corporal". Observou-se uma correlação positiva forte $(\mathrm{p}<0,05)$ entre a idade e o escore do teste FUG. Verificou-se uma correlação negativa moderada da idade com as percepções de condição física $(\mathrm{p}<0,05)$ e habilidade corporal $(\mathrm{p}<$ $0,05)$. Também notou-se uma correlação negativa moderada entre os escores do teste FUG com condição física $(\mathrm{p}<0,05)$ e habilidade corporal $(\mathrm{p}<0,05)$.

Tabela 2 - Resultados da Correlação de Spearman entre idade, FUG, Condição Física, Habilidade Corporal, Saúde e Aparência..

\begin{tabular}{|l|c|c|c|}
\hline \multicolumn{1}{|c|}{ Variáveis } & Estatísticas & Idade & FUG \\
\hline \multirow{2}{*}{ FUG } & $r$ & 0,705 & - \\
\cline { 2 - 4 } & $p$ & $<0,001$ & - \\
\hline \multirow{3}{*}{ CF } & $r$ & $-0,382$ & $-0,482$ \\
\cline { 2 - 4 } & $p$ & 0,045 & 0,009 \\
\hline \multirow{2}{*}{ HC } & $r$ & $-0,558$ & $-0,639$ \\
\cline { 2 - 4 } & $p$ & 0,002 & $<0,001$ \\
\hline \multirow{2}{*}{ S } & $r$ & $-0,165$ & $-0,277$ \\
\cline { 2 - 4 } & $p$ & 0,400 & 0,153 \\
\hline \multirow{2}{*}{$A$} & $r$ & $-0,003$ & 0,218 \\
\cline { 2 - 4 } & $p$ & 0,989 & 0,266 \\
\hline
\end{tabular}

Nota: FUG: Teste "8 foot up-and-go"; CF: Condição física; HC: Habilidade corporal; S: Saúde; A: Aparência.

A Tabela 3 apresenta a matriz de correlação de Spearman entre as categorias da imagem corporal, o teste FUG e as facetas do questionário de qualidade de vida WHOQOL-OLD. Houve uma correlação positiva e moderada entre a percepção de condição física e as facetas Fac 1, Fac 2 e Fac 3 ( $r=0,495)$ do WHOQOL-OLD ( $\mathrm{p}<0,05)$. Fac 6 apesentou uma correlação moderada positiva com a idade ( $p<0,05)$. Também houve correlação moderada e positiva entre o escore do teste FUG com Fac 5 e Fac 6 ( p < 0,05). $\mathrm{O}$ item saúde associou-se de forma moderada e positiva com Fac $4(\mathrm{p}<0,05)$. 
Tabela 3 - Resultados da correlação de Spearman para as seis facetas e qualidade de vida geral do WHOQOL-OLD, idade, FUG, condição física, habilidade corporal, saúde e aparência.

\begin{tabular}{|l|l|l|l|l|l|l|l|}
\hline \multicolumn{1}{|c|}{ Variáveis } & $\begin{array}{l}\text { Estatísti- } \\
\text { cas }\end{array}$ & \multicolumn{1}{|c|}{ Idade } & FUG & CF & HC & S & A \\
\hline \multirow{2}{*}{ Fac1 } & $\mathrm{r}$ & $-0,095$ & $-0,173$ & 0,452 & 0,288 & 0,056 & 0,052 \\
\cline { 2 - 8 } & $\mathrm{p}$ & 0,632 & 0,380 & 0,016 & 0,138 & 0,777 & 0,792 \\
\hline \multirow{2}{*}{ Fac2 } & $\mathrm{r}$ & $-0,279$ & $-0,141$ & 0,472 & 0,238 & 0,258 & 0,103 \\
\cline { 2 - 8 } & $\mathrm{p}$ & 0,151 & 0,474 & 0,011 & 0,223 & 0,185 & 0,600 \\
\hline \multirow{2}{*}{ Fac3 } & $\mathrm{r}$ & $-0,103$ & $-0,074$ & 0,495 & 0,339 & 0,117 & 0,184 \\
\cline { 2 - 8 } & $\mathrm{p}$ & 0,600 & 0,708 & 0,007 & 0,078 & 0,553 & 0,348 \\
\hline \multirow{2}{*}{ Fac4 } & $\mathrm{r}$ & 0,230 & 0,216 & 0,265 & $-0,120$ & 0,385 & 0,293 \\
\cline { 2 - 8 } & $\mathrm{p}$ & 0,240 & 0,269 & 0,172 & 0,544 & 0,043 & 0,131 \\
\hline \multirow{2}{*}{ Fac6 } & $\mathrm{r}$ & 0,267 & 0,395 & 0,040 & $-0,101$ & 0,133 & 0,315 \\
\cline { 2 - 8 } & $\mathrm{p}$ & 0,169 & 0,038 & 0,842 & 0,610 & 0,499 & 0,102 \\
\hline \multirow{2}{*}{ QVGOLD } & $\mathrm{r}$ & 0,399 & 0,419 & 0,116 & $-0,345$ & $-0,023$ & 0,012 \\
\cline { 2 - 8 } & $\mathrm{p}$ & 0,035 & 0,026 & 0,555 & 0,072 & 0,906 & 0,950 \\
\cline { 2 - 8 } & $\mathrm{r}$ & 0,060 & 0,085 & 0,453 & 0,109 & 0,162 & 0,162 \\
\hline
\end{tabular}

Nota: FUG: Teste Foot Up-and-Go; CF: Condição física; HC: Habilidade corporal; S: Saúde; A: Aparência; QVGOLD: Qualidade de vida geral - WHOQOL-OLD

\section{Discussão}

Os resultados do presente apresentaram uma correlação positiva forte entre os escores do teste FUG e a variável idade e uma correlação negativa moderada com as dimensões condição física e habilidade corporal do teste "Minha imagem corporal". Evidências sugerem que classificar a dificuldade de deslocar o corpo como diminuições na condição física e habilidade corporal é uma atitude comum entre idosos que avaliam a sua história de vida pessoal como uma existência auto determinada e com pouca vulnerabilidade na saúde durante a juventude e fase adulta (BAKER; GRINGART, 2009; CAMPBELL; HAUSEMBLAS, 2009). Tal juízo subentende que a experiência vivida constitui um critério para se estabelecer analogias entre a funcionalidade do corpo no passado e no presente conforme a idade avança.

As percepções de condição física e habilidade corporal mensuradas no presente estudo também exibiram correlação negativa moderada com a variável idade. Isso indica que as experiências subjetivas de perda de força, velocidade, agilidade e equilíbrio tornaram-se compreendidas como um processo de progressiva inaptidão e inabilitação do corpo. Embora possuam metodologias distintas, diferentes estudos apresentam resultados que corroboram essa tendência. $\mathrm{O}$ estudo de caso qualitativo efetuado por Tan et al. (2010) com idosos australianos entre 55 e 78 anos de idade 
é um exemplo. Os depoimentos desses sujeitos, analisados por meio da técnica da análise de conteúdo, mostraram que reduções na força e resistência corporais eram algo considerado indesejado porque poderia levá-los a se tornarem um fardo para suas famílias e amigos. Em outro estudo de caso realizado com grupos focais, formados por indivíduos entre 65 e 92 anos no oeste da Inglaterra, Jankowski et al. (2015) registraram que a ausência de restrições motoras severas durante $o$ ato de marchar insurgia como uma preocupação central do processo de envelhecimento, pois a preservação dessa habilidade é que permitia-lhes transitarem com liberdade nos locais públicos. Semelhante tendência comportamental foi verificada em outras pesquisas internacionais conduzidas na China, Japão, Canadá e Estados Unidos (FUNG, 2013; MATSUBAYASHI et al., 2006; HURD-CLARKE, 2001). Em conjunto, tais investigações indicam que estar hábil para caminhar e marchar, assim como sentir-se dotado de força e resistência suficientes, constitui um fator relevante para a vida pessoal.

$\mathrm{O}$ presente estudo identificou ainda uma correlação positiva moderada entre a percepção de condição física e as facetas Fac 1, Fac 2, e Fac 3 do WHOQOL-OLD. Isso significa que quanto mais satisfatória for a aptidão corporal e motora, maior é a valorização dada ao funcionamento íntegro dos órgãos sensoriais; a viver de modo emancipado e ao significado das conquistas passadas e futuras. Tal viés também foi detectado por Molzahn et al. (2010) em estudo com idosos de nações da
América do Sul (Uruguai) e Europa (Turquia) consideradas em desenvolvimento. Todavia, essa tendência não se verificou em idosos residentes nos Estados Unidos da América, Canadá, Japão. Mesmo no Brasil, os resultados das investigações encaminhadas por Paiva et al. (2016) e Campolina et al. (2011) com idosos dos estados de Minas Gerais e São Paulo registraram associações entre a percepção de estar em boa condição física apenas com Fac 1 e Fac 2. Diferenças desta natureza indicam que a vinculação entre a aptidão física e motora do corpo durante o envelhecimento com dimensões da $\mathrm{QV}$ sofre a interferência de particularidades regionais, e culturais (FUNG, 2013; MOLZAHN et al., 2011).

A idade e Fac 6 acusaram uma correlação moderada positiva. Este achado foi parcialmente encontrado no estudo longitudinal de Tetley et al. (2018), conduzido em 2012 e 2013 com 7079 idosos heterossexuais ingleses entre 50 e 90 anos, mostrando-se mais recorrente entre os sujeitos que minimizavam a importância do coito na vida sexual. Esta atitude demonstra que as motivações para a intimidade e amorosidade podem ser modificadas conforme o processo de envelhecimento avança. No entanto, em outro estudo longitudinal realizado com idosos heterossexuais na Inglaterra na mesma faixa etária, Lee et al. (2016) observaram que $39 \%$ dos indivíduos do sexo masculino diziam não ter relacionamentos conjugais satisfatórios devido a disfunções eréteis. Entre as mulheres, a dificuldade estava em serem estimuladas sexualmente (32\%) e no não alcance 
do orgasmo (27\%). Estes idosos também consideravam que tais incapacidades retratavam debilidades na saúde, o que indica que o valor social historicamente atribuído à sexualidade genital pode condicionar as concepções de felicidade na vida conjugal.

Outro resultado importante do presente estudo foi a correlação moderada positiva entre o escore do teste FUG com Fac 5 e Fac 6. Tal associação mostra que comprometimentos na mobilidade corporal ampliam as preocupações dos idosos com a morte e a sua disposição socioafetiva para o companheirismo. Ou seja, a percepção de finitude da vida amplia-se proporcionalmente ao incremento das limitações motoras, condição essa que ocasiona fragilidades emocionais e aumenta o anseio do idoso por auxílios que possam ajudá-lo no controle de suas carências (SOÓSOVÁ, 2016; BILGILI; ARPACI, 2014). Decréscimos progressivos em força, velocidade, equilíbrio, agilidade, resistência e capacidade de deslocamento corporal, quando impactam negativamente os níveis de interesse em viver, são potenciais causadores de ansiedade e depressão (WEBB; WOOD-BARCALOW; TYLKA, 2015). No entanto, o grau de instrução, a religiosidade, medidas psicoterapêuticas e o status socioeconômico podem atenuar essas circunstâncias (SOÓSOVÁ, 2016). Nesse sentido, trabalhos empíricos buscando analisar a interferência relativa de cada uma dessas variáveis no enfrentamento das debilidades físicas e mentais carecem de ser endereçados (LAYTE; SEXTON; SAVVA, 2013).
O presente estudo mostrou uma associação moderada e positiva entre a dimensão saúde da imagem corporal e a Fac 4, ratificando que o idoso aparentemente saudável é mais propenso a buscar contatos sociais. Resultados similares foram encontrados em pesquisas de Chin, Lee \& Lee (2014) e Forjaz et al. (2015), que descobriram pioras na participação de idosos em atividades culturais e socializáveis (cultos religiosos, espetáculos esportivos, passeios etc.) em consequência do agravamento de morbidades cardiovasculares, osteomioarticulares e mentais. Logo, a construção de relacionamentos sociais e a execução de atividades da vida diária dependem do quanto o idoso se vê como saudável.

$\mathrm{O}$ presente estudo apresentou limitações em seu delineamento, como o fato de ser uma investigação de natureza transversal, o que não permite analisar variações da associação entre autoimagem, mobilidade física e facetas da qualidade de vida à medida que o tempo transcorre. Além disso, a amostra limitou-se a critérios de conveniência, incluindo apenas os indivíduos participantes de um mesmo programa de atividades físicas. Entretanto, seus resultados são relevantes para a melhor compreensão do papel da prática regular de exercícios físicos junto a idosos, que vão para além de seus benefícios biológicos já amplamente divulgados. Percebe-se que o impacto dessa prática pode contribuir para o bem-estar geral dessa população, incluindo aspectos psicossociais importantes para que os seres 
humanos possam aproveitar o maior tempo de vida possível com qualidade.

\section{Conclusão}

No presente estudo, o avanço cronológico da idade está associado com retardos na capacidade motora corporal, os quais acabaram subjetivamente percebidos como menores condição física e habilidade corporal. Por sua vez, a percepção do idoso de estar condicionado fisicamente, a sua mobilidade corporal e a idade cronológica exerceram interferência em todas as facetas da qualidade de vida do WHOQOL-Old, porém, em diferentes proporções.

Como sugestões para futuras pesquisas, recomenda-se a efetivação de trabalhos longitudinais, na medida em que possibilitam averiguar como estas variáveis comportam-se temporalmente.

\section{Associations between body mobility, self-image and quality of life in elderly individuals integrated in a systematic physical activity program}

\section{Abstract}

The aim of the present study was to investigate the associations between physical mobility, body images and quality of life perception in elderly individuals taking part a systematic program of physical activities. The sample consisted of 28 elderly men and women (16 men and 12 women). The instruments for data collection were the 8 foot up-and-go (FUG) test, the My Body Image questionnaire and the WHOQOL-Old Quality of Life questionnaire. Data were treated using descriptive and correlational statistics. Spearman's correlation test showed a positive correlation between the age of the elderly and the FUG test score ( $p$ $<0.001)$. Positive and significant correlations were also detected between the perception of physical condition and WHOQOL-OLD Fac 1, Fac 2 and Fac 3 facets ( $p$ $<0.05)$; Fac 6 and age $(p<0.05)$; FUG test score and Fac 5 and Fac $6(p<0.05)$; health perception and Fac $4(p<0.05)$. There were significant and negative correlations between age and perceptions of physical condition and body skills ( $p<0.05)$; FUG test score and perception of physical condition and body skills $(p<0.05)$. The study concluded that the delay in motor capacity due to aging is associated with reduced physical condition and body skills, as well as the perception of the elderly about fitness, their body mobility and chronological age may interfere in the facets of quality of life through different proportions.

Keywords: Elderly. Body mobility. Self-image. Quality of life. Physical activity.

\section{Referências}

BAKER, L.; GINGART, E. Body image and self-esteem in older adulthood. Aging \& Society, v. 29, n. 6, p. 977-995, 2009.

BILGILI, N.; ARPACI, F. Quality of life in older adults in Turkey. Archives of Gerontology and Geriatrics; Amsterdam, n. 59, v. 2, p. 415-421, 2014.

BORELLA, E. et al. Spatial abilities across de adult life span. Developmental Psychology, n. 50 , v. 2, 384-392, 2004.

CAMPBELL, A.; HAUSEMBLAS, H. A. Effects of exercise interventions on body image: a meta-analysis. Journal of Health Psychology, v. 14, n. 6, p. 780-793, 2009. 
CAMPOLINA, A. G.; DINI, P.S.; CICONELLI, R. M. Impacto da doença crônica na qualidade de vida de idosos da comunidade em São Paulo (SP, Brasil). Ciência \& Saúde Coletiva, Rio de Janeiro, v. 16, n. 6, p. 29192925, 2011.

CHAN, W. C. et al. Efficacy of physical exercise in preventing falls in older adults with cognitive impairment: a systematic review and meta-analysis. Journal of the American Medical Directors Association, v. 16, n. 2, p. 149-54, 2015.

CHAO, Y. Y.; SCHERER, Y. K.; MONTGOMERY, C. A. Effects of Using Nintendo WiiTM Exergames in Older Adults: A Review of the Literature. Journal of Aging and Health, v. 27, n. 3, p. 379-402, 2015.

CHIN, Y. R.; LEE, I. S.; LEE, H. Y. Effects of hypertension, diabetes, and/or cardiovascular disease on health-related quality of life in elderly Korean individuals: a population-based cross-sectional survey. Asian Nursing Research, v. 8, n. 4, p. 267-273, 2014.

FLECK, M. P. A, CHACHAMOVICH, E.; RENTINI, C. M. Projeto WHOQOL-OLD: método e resultados de grupos focais no Brasil. Revista de Saúde Pública, São Paulo, v. 37, n. 6, p. 793-799, 2003.

FORJAZ, M. J. et. al. Chronic conditions, disability, and quality of life in older adults with multimorbidity in Spain. European Journal of Internal Medicine, v. 26, n. 3, p.176-181, 2015.

FUNG, H. H. Aging in Culture. The Gerontologist, v. 53, n. 3, p. 369-377, 2013.

GANESH, K. S.; ANINDO, M.; PAVITHRA, G. Quality of Life (QOL) and Its Associated Factors Using WHOQOL-BREF Among Elderly in Urban Puducherry, India. Journal of Clinical and Diagnostic Research, v. 8, n. 1, p. 54-57, 2014.

GOMES, M. M. et al. Analysis of postural control and muscular performance in young and elderly women in different age groups.
Brazilian Journal of Physical Therapy, São Carlos, v. 19, n. 1, p.1-9, 2015.

HURD-CLARKE, L. Older women's bodies and the self: The construction of identity in later life. Canadian Review of Sociology and Anthropology, v. 38, n. 4, p. 441-461, 2001.

INOUYE, K. et al. Efeito da Universidade Aberta à Terceira Idade sobre a qualidade de vida do idoso. Educação e Pesquisa, São Paulo, v. 44, e142931, 2018.

JANKOWSKI, G. S. et al. Looking age-appropriate while growing old gracefully: A qualitative study of ageing and body image among older adults. Journal of Health Psychology, v.21, n. 4, p. 550-561, 2016.

LAM, F. M. H. et al. Physical exercise improves strength, balance, mobility, and endurance in people with cognitive impairment and dementia: a systematic review. Journal of Physiotherapy, v. 64, n. 1, p. 4-15, 2018.

LAYTE, R.; SEXTON, E.; SAVVA, G. Quality of life in older age: evidence from an Irisch cohort study. Journal of the American Geriatrics Society, v. 61, n. 2, p. 299-305, 2013.

LEE, D. M. et al. Sexual health and wellbeing among older men and women in England: findings from the English Longitudinal Study of Ageing. Archives of Sexual Behavior, v. 45, n.1, p. 133-144, 2016.

LOVO, T. M. A. Adaptação e aplicação de questionários de imagem corporal em portadores de hemiplegia. 2001. Monografia (Monografia de Especialização em Educação Física Adaptada). Faculdade de Educação Física, Universidade Estadual de Campinas, Campinas, 2001.

MATSUBAYASHI, K. et al. Older adults' views of "successful aging": comparison of older Japanese and Americans. Journal of the American Geriatrics Society, v. 54, n. 1, p. 184-187, 2006.

MOLZAHN, A. et al. The importance of facets of quality of life to older adults: an interna- 
tional investigation. Quality of Life Research, Oxford, v. 19, n. 2, p. 293-298, 2010.

MOLZAHN, A. et al. Comparing the importance of different aspects of quality of life to older adults across diverse cultures. Age and Ageing, v. 40, n. 2, p. 192-199, 2011.

OSWALD, W. D. et al. Differential effects of single versus combined cognitive and physical training with older adults: the SimA study in a 5-year perspective. European Journal of Ageing, v.10, n. 3, p. 179-192, 2006.

PAIVA, M. H. P. et al. Fatores associados à qualidade de vida de idosos comunitários da macrorregião do Triângulo do Sul, Minas Gerais, Brasil. Ciência \& Saúde Coletiva, Rio de Janeiro, v. 21, n. 11, p. 3347-3356, 2016.

PITKÄLÄ, K. et al. Efficacy of physical exercise intervention on mobility and physical functioning in older people with dementia: a systematic review. Experimental Geronto$\log y$, v. 48, n. 1, p. 85-93, 2013.

RIKLI, R.; JONES, C. Development and validation of a functional fitness test for community-residing older adults. Journal of Aging and Physical Activity, v. 7, n. 2, p. 129-161, 1999.

SANTOS, P. L.; FORONI, P. M.; CHAVES, M. C. F. Atividades físicas e de lazer e seu impacto sobre a cognição no envelhecimento. Medicina, Ribeirão Preto, v. 42, n. 1, p. 5460, 2009.

SOÓSOVÁ, M. S. Determinants of quality of life in the elderly. Central European Journal of Nursery and Midwifery, v. 7, n.3, p. 484493, 2016.

TAÍROVA, O. S.; LORENZ, D. R. S. Influência do exercício físico na qualidade de vida de mulheres na pós-menopausa: um estudo caso-controle. Revista Brasileira de Geriatria e Gerontologia, Rio de Janeiro, v. 14, n. 1, p. 135-145, 2011.

TAN, J.; WARD, L.; ZIAIAN, T. Experiences of Chinese immigrants and Anglo-Australians ageing in Australia: a cross-cultural perspective on successful ageing. Journal of Health Psychology, v. 15, n. 5, p. 697-706, 2010 .

TETLEY, J. et al. Let's talk about sex - what do older men and women say about their sexual relations and sexual activities? A qualitative analysis of ELSA Wave 6 data. Ageing \& Society, v. 38, n. 3, p.497-521, 2018.

TYLKA, T. L.; WOOD-BARCALOW, N. L. What is and what is not positive body image? Conceptual foundations and construct definition. Body Image, v. 14, p. 118-129, 2015.

VALE, R. G. S et al. Effects of resistance training and chess playing on the quality of life and cognitive performance of elderly women: a randomized controlled trial. Journal of Physical Education and Sport, v. 18, n. 3, p. 1469-1477, 2018.

WEBB, J. B.; WOOD-BARCALOW, N. L.; TYLKA, T. L. Assessing positive body image: Contemporary approaches and future directions. Body Image, v. 14, p. 130-145, 2015.

ZIELINSKA-WIECZKOWSKA, H. Correlations between satisfaction with life and selected personal resources among students of Universities of the Third Age. Clinical Interventions in Aging, v. 12, p. 1391-1399, 2017. 